\title{
Uniform Estimates for Damped Radon Transform on the Plane
}

\author{
Youngwoo Choi \\ Department of Mathematics, Ajou University, Suwon 443-749, Republic of Korea \\ Correspondence should be addressed to Youngwoo Choi; ychoi100@gmail.com
}

Received 13 May 2013; Accepted 22 August 2013

Academic Editor: Wenchang Sun

Copyright (c) 2013 Youngwoo Choi. This is an open access article distributed under the Creative Commons Attribution License, which permits unrestricted use, distribution, and reproduction in any medium, provided the original work is properly cited.

Uniform improving estimates of damped plane Radon transforms in Lebesgue and Lorentz spaces are studied under mild assumptions on the rotational curvature. The results generalize previously known estimates. Also, they extend sharp estimates known for convolution operators with affine arclength measures to the semitranslation-invariant case.

\section{Introduction}

Let $\mathscr{D}$ be a domain in $\mathbb{R}^{2}$ and let

$$
\begin{aligned}
& \mathscr{D}^{*}:=\left\{x_{1} \in \mathbb{R}:\left(x_{1}, y\right) \in \mathscr{D} \text { for some } y \in \mathbb{R}\right\}, \\
& \mathscr{D}_{*}:=\left\{y \in \mathbb{R}:\left(x_{1}, y\right) \in \mathscr{D} \text { for some } x_{1} \in \mathbb{R}\right\} .
\end{aligned}
$$

For $x_{1} \in \mathscr{D}^{*}$ and $y \in \mathscr{D}_{*}$, we write

$$
\begin{gathered}
\mathscr{D}_{x_{1}}:=\left\{z \in \mathbb{R}:\left(x_{1}, z\right) \in \mathscr{D}\right\}, \\
\mathscr{D}^{y}:=\{z \in \mathbb{R}:(z, y) \in \mathscr{D}\} .
\end{gathered}
$$

To avoid technical difficulties, we assume that, for $x_{1} \in \mathscr{D}^{*}$ and $y \in \mathscr{D}_{*}, \mathscr{D}_{x_{1}}$ and $\mathscr{D}^{y}$ are finite intervals throughout the paper. For a $C^{2}$ function $\varphi$ and a measurable function $\omega$ defined on $\mathscr{D}$, we consider the damped Radon transform $\mathscr{R}_{\varphi, \omega}$ defined by

$$
\mathscr{R}_{\varphi, \omega} f\left(x_{1}, x_{2}\right):=\int_{\mathscr{D}_{x_{1}}} f\left(y, x_{2}+\varphi\left(x_{1}, y\right)\right) \omega\left(x_{1}, y\right) d y
$$

for $f \in C_{0}^{\infty}\left(\mathbb{R}^{2}\right)$. Mapping properties of such operators in various function spaces have been studied by many authors [1-9]. Sharper estimates are available in translation-invariant cases where $\varphi\left(x_{1}, y\right)=\phi\left(x_{1}-y\right)$ with a $C^{2}$ function $\phi$ defined on an interval $[10,11]$ and it is widely known that the so-called affine arclength measure introduced by Drury [12] is better suited in obtaining degeneracy independent results in many interesting cases. Analogous quantity in nontranslation-invariant situation is rotational curvature, which is given by $\varphi_{12}^{\prime \prime}\left(x_{1}, y\right)=\partial^{2} \varphi / \partial x_{1} \partial y$ in this setting. In this paper, we are interested in uniform optimal improving properties in Lebesgue spaces and Lorentz spaces. The results will generalize known estimates for damped Radon transform and convolution operators with affine arclength measure on plane curves.

Before we state the results, we introduce certain conditions on functions defined on intervals. For an interval $J_{1}$ in $\mathbb{R}$, a locally integrable function $\Phi: J_{1} \rightarrow \mathbb{R}^{+}$, and a positive real number $A$, we let

$$
\begin{gathered}
\mathfrak{G}(\Phi, A):=\left\{\omega: J_{1} \longrightarrow \mathbb{R}^{+} \mid \sqrt{\omega\left(s_{1}\right) \omega\left(s_{2}\right)}\right. \\
\leq \frac{A}{s_{2}-s_{1}} \int_{s_{1}}^{s_{2}} \Phi(s) d s \\
\text { whenever } \left.s_{1}<s_{2} \text { and }\left[s_{1}, s_{2}\right] \subset J_{1}\right\}, \\
\mathscr{E}_{1}(A):=\left\{\Phi: J \longrightarrow \mathbb{R}^{+} \mid \Phi \in \mathfrak{G}(\Phi, A)\right\} .
\end{gathered}
$$

An interesting subclass of $\mathscr{E}_{1}(2 A)$ is the collection $\mathscr{E}_{2}(A)$, introduced in [13], of functions $\Phi: J \rightarrow \mathbb{R}^{+}$such that

(1) $\Phi$ is monotone,

(2) whenever $s_{1}<s_{2}$ and $\left[s_{1}, s_{2}\right] \subset J$,

$$
\sqrt{\Phi\left(s_{1}\right) \Phi\left(s_{2}\right)} \leq A \Phi\left(\frac{s_{1}+s_{2}}{2}\right) .
$$


In connection with the problems related to convolution operators with affine arclength measure on curves in the plane, the author of [10] proved the following.

Theorem 1. Let $J$ be an open interval in $\mathbb{R}$, and let $\phi: J \rightarrow \mathbb{R}$ be a $C^{2}$ function such that $\phi^{\prime \prime} \geq 0$. Let $\omega: J \rightarrow \mathbb{R}$ be a nonnegative measurable function. Suppose that there exists a positive constant $A$ such that $\omega \in \mathfrak{G}\left(\phi^{\prime \prime}, A\right)$; that is,

$$
\omega\left(s_{1}\right)^{1 / 2} \omega\left(s_{2}\right)^{1 / 2} \leq \frac{A}{s_{2}-s_{1}} \int_{s_{1}}^{s_{2}} \phi^{\prime \prime}(v) d v
$$

holds whenever $s_{1}<s_{2}$ and $\left[s_{1}, s_{2}\right] \subset J$. Let $\mathcal{S}_{\phi}$ be the operator given by

$$
\mathcal{\delta}_{\phi} f\left(x_{1}, x_{2}\right)=\int_{J} f\left(x_{1}-s, x_{2}-\phi(s)\right) \omega^{1 / 3}(s) d s
$$

for $f \in C_{0}^{\infty}\left(\mathbb{R}^{2}\right)$. Then, there exists a constant $C$ that depends only on $A$ such that

$$
\left\|\mathcal{S}_{\phi} f\right\|_{L^{3}\left(\mathbb{R}^{2}\right)} \leq C\|f\|_{L^{3 / 2}\left(\mathbb{R}^{2}\right)}
$$

holds uniformly in $f \in C_{0}^{\infty}\left(\mathbb{R}^{2}\right)$.

Regarding the endpoint Lorentz space estimates, the following result due to Oberlin is available.

Theorem 2 (Oberlin [11]). Let $\phi$ be a $C^{2}$ function on an interval J such that $\phi^{\prime \prime}>0$ on $J$ and $\phi^{\prime \prime} \in \mathscr{E}_{2}(A)$. Then, $\mathcal{S}_{\phi}$ defined in (7) maps $L^{3 / 2,3}\left(\mathbb{R}^{2}\right)$ boundedly to $L^{3}\left(\mathbb{R}^{2}\right)$ with the operator norm depending only on $A$.

In this paper, the author generalizes the aforementioned theorems to damped Radon transforms where the condition on the affine arclength measure is replaced by that on the rotational curvature. This paper is organized as follows: in Section 2, uniform estimate in Lebesgue spaces is studied, and in Section 3, endpoint Lorentz space estimate will be given based on an approach similar to Oberlin's approach $[11,14]$.

\section{Uniform Estimates on the Plane}

Theorem 3. Let $\varphi$ be a $C^{2}$ function on $\mathscr{D}$ such that $\varphi_{12}^{\prime \prime}>0$, and let $\omega$ be a nonnegative measurable function on $\mathscr{D}$. Suppose that there exists a positive constant $A$ such that, for each $x_{1} \in$ $\mathscr{D}^{*}, \omega\left(x_{1}, \cdot\right) \in \mathfrak{G}\left(\varphi_{12}^{\prime \prime}\left(x_{1}, \cdot\right), A\right)$; that is,

$$
\omega\left(x_{1}, y_{1}\right)^{1 / 2} \omega\left(x_{1}, y_{2}\right)^{1 / 2} \leq \frac{A}{y_{2}-y_{1}} \int_{y_{1}}^{y_{2}} \varphi_{12}^{\prime \prime}\left(x_{1}, z\right) d z
$$

holds whenever $y_{1}<y_{2}$ and $\left[y_{1}, y_{2}\right] \subset \mathscr{D}_{x_{1}}$. Let $\mathscr{R}_{\varphi, \omega}$ be the operator given by (3). Then, there exists a constant $C$ that depends only on A such that

$$
\left\|\mathscr{R}_{\varphi, \omega} f\right\|_{L^{3}\left(\mathbb{R}^{2}\right)} \leq C\|f\|_{L^{3 / 2}\left(\mathbb{R}^{2}\right)}
$$

holds uniformly in $f \in C_{0}^{\infty}\left(\mathbb{R}^{2}\right)$.
Proof of Theorem 3. Our proof is based on the method introduced by Drury and Guo [15], which was later refined by Oberlin [16] and the author of [10]. We have

$$
\begin{gathered}
\left\|\mathscr{R}_{\varphi, \omega} f\right\|_{3}^{3} \\
=\int_{\mathbb{R}} \int_{\mathscr{D}^{*}} \prod_{j=1}^{3}\left(\int_{\mathscr{D}_{x_{1}}} f\left(y_{1}, x_{2}+\varphi\left(x_{1}, y_{j}\right)\right)\right. \\
\left.\times \omega^{1 / 3}\left(x_{1}, y_{j}\right) d y_{j}\right) d x_{1} d x_{2} \\
=\iiint_{\mathscr{D}_{*}}\left[\mathscr{G}\left(f\left(y_{1}, \cdot\right), f\left(y_{2}, \cdot\right), f\left(y_{3}, \cdot\right)\right)\right] \\
\quad \times\left(y_{1}, y_{2}, y_{3}\right) d y_{1} d y_{2} d y_{3},
\end{gathered}
$$

where for $y_{1}, y_{2}, y_{3} \in \mathscr{D}_{*}$ and suitable functions $g_{1}, g_{2}, g_{3}$ defined on $\mathbb{R}$,

$$
\begin{aligned}
& {\left[\mathscr{G}\left(g_{1}, g_{2}, g_{3}\right)\right]\left(y_{1}, y_{2}, y_{3}\right)} \\
& :=\int_{\mathbb{R}} \int_{\mathscr{D}_{1}, y_{2}, y_{3}} \prod_{j=1}^{3}\left[g_{j}\left(x_{2}+\varphi\left(x_{1}, y_{j}\right)\right) \omega^{1 / 3}\left(x_{1}, y_{j}\right)\right] d x_{1} d x_{2}
\end{aligned}
$$

with $\mathscr{D}^{y_{1}, y_{2}, y_{3}}:=\bigcap_{j=1}^{3} \mathscr{D}^{y_{j}}$. As in the proof of Theorem $2.1 \mathrm{in}$ [10], one can show that the estimate

$$
\begin{aligned}
& \left|\left[\mathscr{G}\left(g_{1}, g_{2}, g_{3}\right)\right]\left(y_{1}, y_{2}, y_{3}\right)\right| \\
& \quad \leq \frac{C\left\|g_{1}\right\|_{L^{3 / 2}(\mathbb{R})}\left\|g_{2}\right\|_{L^{3 / 2}(\mathbb{R})}\left\|g_{3}\right\|_{L^{3 / 2}(\mathbb{R})}}{\left|\left(y_{1}-y_{2}\right)\left(y_{1}-y_{3}\right)\left(y_{2}-y_{3}\right)\right|^{1 / 3}}
\end{aligned}
$$

holds uniformly in $g_{1}, g_{2}, g_{3}, y_{1}, y_{2}$, and $y_{3}$. Combining this with Proposition 2.2 in the work by Christ [17] finishes the proof.

Remark 4. The special case in which $\omega=\varphi_{12}^{\prime \prime}$ provides a uniform estimate for the damped plane Radon transform. We write

$\mathscr{R}_{\varphi} f\left(x_{1}, x_{2}\right)=\int_{\mathscr{D}_{x_{1}}} f\left(y, x_{2}+\varphi\left(x_{1}, y\right)\right) \varphi_{12}^{\prime \prime}\left(x_{1}, x_{2}, y\right)^{1 / 3} d y$

for $f \in C_{0}^{\infty}\left(\mathbb{R}^{2}\right)$.

Corollary 5. Let $\varphi: \mathscr{D} \rightarrow \mathbb{R}$ be a $C^{2}$ function such that $\varphi_{12}^{\prime \prime}>0$. Suppose that there exists a constant A such that, for each $x_{1} \in \mathscr{D}^{*}, \varphi_{12}^{\prime \prime}\left(x_{1}, \cdot\right) \in \mathscr{E}_{1}(A)$; that is,

$$
\varphi_{12}^{\prime \prime}\left(x_{1}, y_{1}\right)^{1 / 2} \varphi_{12}^{\prime \prime}\left(x_{1}, y_{2}\right)^{1 / 2} \leq \frac{A}{y_{2}-y_{1}} \int_{y_{1}}^{y_{2}} \varphi_{12}^{\prime \prime}\left(x_{1}, z\right) d z
$$

holds whenever $y_{1}<y_{2}$ and $\left[y_{1}, y_{2}\right] \subset \mathscr{D}_{x_{1}}$. Let $\mathscr{R}_{\varphi}$ be the operator given by (14). Then, there exists a constant $C$ that depends only on A such that

$$
\left\|\mathscr{R}_{\varphi} f\right\|_{L^{3}\left(\mathbb{R}^{2}\right)} \leq C\|f\|_{L^{3 / 2}\left(\mathbb{R}^{2}\right)}
$$

holds uniformly in $f \in C_{0}^{\infty}\left(\mathbb{R}^{2}\right)$. 
Remark 6. A duality argument shows the following.

Corollary 7. Let $\varphi: \mathscr{D} \rightarrow \mathbb{R}$ be a $C^{2}$ function such that $\varphi_{12}^{\prime \prime}>0$. Suppose that there exists a constant A such that, for each $y_{1} \in \mathscr{D}_{*}, \varphi_{12}^{\prime \prime}\left(\cdot, y_{1}\right) \in \mathscr{E}_{1}(A)$; that is,

$$
\varphi_{12}^{\prime \prime}\left(x_{1}, y_{1}\right)^{1 / 2} \varphi_{12}^{\prime \prime}\left(x_{2}, y_{1}\right)^{1 / 2} \leq \frac{A}{x_{2}-x_{1}} \int_{x_{1}}^{x_{2}} \varphi_{12}^{\prime \prime}\left(z, y_{1}\right) d z
$$

holds whenever $x_{1}<x_{2}$ and $\left[x_{1}, x_{2}\right] \subset \mathscr{D}^{y_{1}}$. Let $\mathscr{R}_{\varphi}$ be the operator given by (14). Then, there exists a constant $C$ that depends only on A such that

$$
\left\|\mathscr{R}_{\varphi} f\right\|_{L^{3}\left(\mathbb{R}^{2}\right)} \leq C\|f\|_{L^{3 / 2}\left(\mathbb{R}^{2}\right)}
$$

holds uniformly in $f \in C_{0}^{\infty}\left(\mathbb{R}^{2}\right)$.

\section{Endpoint Lorentz Estimates}

Under somewhat stronger condition, estimates in Section 2 can be improved. Namely, we have the following.

Theorem 8. Let $\varphi: \mathscr{D} \rightarrow \mathbb{R}$ be a $C^{2}$ function such that $\varphi_{12}^{\prime \prime}>$ 0 . Suppose that there exists a constant $A$ such that, for each $x_{1} \in \mathscr{D}^{*}, \varphi_{12}^{\prime \prime}\left(x_{1}, \cdot\right) \in \mathscr{E}_{2}(A)$; that is,

$$
\varphi_{12}^{\prime \prime}\left(x_{1}, y_{1}\right)^{1 / 2} \varphi_{12}^{\prime \prime}\left(x_{1}, y_{2}\right)^{1 / 2} \leq A \varphi_{12}^{\prime \prime}\left(x_{1}, \frac{\left(y_{1}+y_{2}\right)}{2}\right)
$$

holds whenever $y_{1}<y_{2}$ and $\left[y_{1}, y_{2}\right] \subset \mathscr{D}_{x_{1}}$. Let $\mathscr{R}_{\varphi}$ be the operator given by (14). Then, there exists a constant $C$ that depends only on A such that

$$
\left\|\mathscr{R}_{\varphi} f\right\|_{L^{3,3 / 2}\left(\mathbb{R}^{2}\right)} \leq C\|f\|_{L^{3 / 2}\left(\mathbb{R}^{2}\right)}
$$

holds uniformly in $f \in C_{0}^{\infty}\left(\mathbb{R}^{2}\right)$.

Proof of Theorem 8. To ease our notation, we let $\omega:=\varphi_{12}^{\prime \prime}$. For a measurable subset $E$ of either $\mathbb{R}$ or $\mathbb{R}^{2}$, we denote the Lebesgue measure and the characteristic function of $E$ by $|E|$ and $\mathbb{1}_{E}$, respectively.

By a well-known interpolation argument as in $[2,18]$, it suffices to establish the estimate

$$
\begin{aligned}
I\left(E_{1}, E_{2}, E_{3}\right) & :=\iint_{\mathbb{R}^{2}} \prod_{j=1}^{3}\left[\mathscr{R} \mathbb{1}_{E_{j}}\left(x_{1}, x_{2}\right)\right] d x_{1} d x_{2} \\
& \leq C\left|E_{1}\right|\left|E_{2}\right|^{1 / 2}\left|E_{3}\right|^{1 / 2}
\end{aligned}
$$

for all measurable subsets $E_{1}, E_{2}$, and $E_{3}$ of $\mathbb{R}^{2}$. We have

$$
\begin{aligned}
& I\left(E_{1}, E_{2}, E_{3}\right) \\
& =\iint_{\mathbb{R}^{2}} \prod_{j=1}^{3}\left[\int_{\mathscr{D}_{x_{1}}} \mathbb{1}_{E_{j}}\left(y_{j}, x_{2}+\varphi\left(x_{1}, y_{j}\right)\right)\right. \\
& \left.\times \omega\left(x_{1}, y_{j}\right)^{1 / 3} d y_{j}\right] d x_{1} d x_{2} \\
& =\int_{\mathscr{D}_{*}} \int_{\mathbb{R}}\left[\mathscr{A}\left(x_{2}, y_{1} ; E_{2}, E_{3}\right)\right] \mathbb{1}_{E_{1}}\left(y_{1}, x_{2}\right) d x_{2} d y_{1},
\end{aligned}
$$

where

$$
\begin{aligned}
& \mathscr{A}\left(x_{2}, y_{1} ; E_{2}, E_{3}\right) \\
& \quad:=\int_{\mathscr{D}^{y_{1}}} \prod_{j=2}^{3}\left[\widetilde{\mathscr{B}}\left(x_{1}, x_{2}, y_{1} ; E_{j}\right)\right] \omega\left(x_{1}, y_{1}\right)^{1 / 3} d x_{1}, \\
& \widetilde{\mathscr{B}}\left(x_{1}, x_{2}, y_{1} ; E\right) \\
& \quad:=\int_{\mathscr{D}_{x_{1}}} \mathbb{1}_{E}\left(y, x_{2}+\varphi\left(x_{1}, y\right)-\varphi\left(x_{1}, y_{1}\right)\right) \omega\left(x_{1}, y\right)^{1 / 3} d y .
\end{aligned}
$$

By Schwarz inequality, it suffices to get an estimate

$$
\int_{\mathscr{D}^{y_{1}}}\left[\widetilde{\mathscr{B}}\left(x_{1}, x_{2}, y_{1} ; E\right)\right]^{2} \omega\left(x_{1}, y_{1}\right)^{1 / 3} d x_{1} \leq C|E|
$$

uniformly in $x_{2}, y_{1}$, and $E$. By translation invariance of $\widetilde{\mathscr{B}}\left(x_{1}, x_{2}, y_{1} ; E\right)$ in $x_{2}$ variable, it is enough to establish

$$
\begin{gathered}
\int_{\mathscr{D}^{y_{1}}}\left[\mathscr{B}_{1}\left(x_{1}, y_{1} ; E\right)\right]^{2} \omega\left(x_{1}, y_{1}\right)^{1 / 3} d x_{1} \leq C|E|, \\
\int_{\mathscr{D}^{y_{1}}}\left[\mathscr{B}_{2}\left(x_{1}, y_{1} ; E\right)\right]^{2} \omega\left(x_{1}, y_{1}\right)^{1 / 3} d x_{1} \leq C|E|
\end{gathered}
$$

uniformly in $y_{1}$ and $E$, where

$$
\begin{aligned}
& \mathscr{B}_{1}\left(x_{1}, y_{1} ; E\right) \\
& \quad:=\int_{\mathscr{D}_{x_{1}} \cap\left[y_{1}, \infty\right)} \mathbb{1}_{E}\left(y, \varphi\left(x_{1}, y\right)-\varphi\left(x_{1}, y_{1}\right)\right) \omega\left(x_{1}, y\right)^{1 / 3} d y, \\
& \mathscr{B}_{2}\left(x_{1}, y_{1} ; E\right) \\
& \quad:=\int_{\mathscr{D}_{x_{1}} \cap\left(-\infty, y_{1}\right)} \mathbb{1}_{E}\left(y, \varphi\left(x_{1}, y\right)-\varphi\left(x_{1}, y_{1}\right)\right) \omega\left(x_{1}, y\right)^{1 / 3} d y .
\end{aligned}
$$

Notice that the map $\Gamma:\left(x_{1}, y\right) \mapsto\left(y, \varphi\left(x_{1}, y\right)-\varphi\left(x_{1}, y_{1}\right)\right)$ is one-to-one and has the absolute value of Jacobian determinant $J\left(x_{1}, y\right):=\left|\varphi_{1}^{\prime}\left(x_{1}, y\right)-\varphi_{1}^{\prime}\left(x_{1}, y_{1}\right)\right|$ for a given $y_{1} \in \mathscr{D}_{*}$.

3.1. Estimate for $\mathscr{B}_{1}$. We follow an approach by Oberlin [14]. Letting

$$
F:=F\left(x_{1}, y_{1} ; E\right):=\left\{y \in \mathscr{D}_{x_{1}} \cap\left[y_{1}, \infty\right): \Gamma\left(x_{1}, y\right) \in E\right\}
$$

we have

$$
\begin{aligned}
& \int_{\mathscr{D}_{x_{1}} \cap\left[y_{1}, \infty\right)} \mathbb{1}_{F}(y)\left(\varphi_{1}^{\prime}\left(x_{1}, y\right)-\varphi_{1}^{\prime}\left(x_{1}, y_{1}\right)\right) d y \\
& \quad=\int_{\mathscr{D}_{x_{1}} \cap\left[y_{1}, \infty\right)} \mathbb{1}_{F}(y) \int_{y_{1}}^{y} \omega\left(x_{1}, z\right) d z d y \\
& \quad=\int_{\left[y_{1}, \infty\right)}\left|F_{z}\right| \omega\left(x_{1}, z\right) d z .
\end{aligned}
$$


Here, for $z>y_{1}$, we denoted by $F_{z}$ the set $F \cap[z, \infty)$. On the other hand, applying Hölder's inequality as in [14], we get

$$
\begin{aligned}
& \int_{\mathscr{D}_{x_{1}} \cap\left[y_{1}, \infty\right)} \mathbb{1}_{F}(z) \omega\left(x_{1}, z\right)^{1 / 3} d z \\
& \leq 2^{2 / 3}|F|^{1 / 3}\left(\int_{\mathscr{D}_{x_{1}} \cap\left[y_{1}, \infty\right)} \mathbb{1}_{F}(y) J\left(x_{1}, y\right) d y\right)^{1 / 3} .
\end{aligned}
$$

Combined with the monotonicity of $\omega\left(x_{1}, \cdot\right)$, we obtain

$$
\begin{aligned}
& \omega\left(x_{1}, y_{1}\right)^{1 / 3}\left[\mathscr{B}_{1}\left(x_{1}, y_{1} ; E\right)\right]^{2} \\
& \quad=\omega\left(x_{1}, y_{1}\right)^{1 / 3}\left(\int_{\mathscr{D}_{x_{1}} \cap\left[y_{1}, \infty\right)} \mathbb{1}_{F}(z) \omega\left(x_{1}, z\right)^{1 / 3} d z\right)^{2} \\
& \leq|F|^{-1}\left(\int_{\mathscr{D}_{x_{1}} \cap\left[y_{1}, \infty\right)} \mathbb{1}_{F}(z) \omega\left(x_{1}, z\right)^{1 / 3} d z\right)^{3} \\
& \leq 4 \int_{\mathscr{D}_{x_{1}} \cap\left[y_{1}, \infty\right)} \mathbb{1}_{F}(y) J\left(x_{1}, y\right) d y .
\end{aligned}
$$

An integration in $x_{1}$ provides (25).

3.2. Estimate for $\mathscr{B}_{2}$. For fixed $x_{1}$ and $y_{1}$, we let

$$
\rho=\frac{1}{2 C(1)} \int_{\mathscr{D}_{x_{1}} \cap\left(-\infty, y_{1}\right)} \mathbb{1}_{F}(y) \omega\left(x_{1}, y\right)^{1 / 3} d y,
$$

where $C(1)$ is the constant that appears in Lemma 2.2 in [11], which implies

$$
\begin{aligned}
& \int_{\omega\left(x_{1}, y_{1}\right)^{1 / 3}\left|y-y_{1}\right| \geq \rho} \mathbb{1}_{F}(y) \omega\left(x_{1}, y\right)^{1 / 3} d y \\
& \quad \geq \frac{1}{2} \int_{\mathscr{D}_{x_{1}} \cap\left(-\infty, y_{1}\right)} \mathbb{1}_{F}(y) \omega\left(x_{1}, y\right)^{1 / 3} d y .
\end{aligned}
$$

Since $\omega\left(x_{1}, \cdot\right)$ is nondecreasing, we see

$$
\begin{aligned}
& \text { LHS of (26) } \leq 2 \int_{\mathscr{D}^{*}} \int_{\mathscr{D}_{x_{1}} \cap\left(-\infty, y_{1}\right)}\left|y_{1}-y\right| \omega\left(x_{1}, y_{1}\right)^{2 / 3} \\
& \quad \times \omega\left(x_{1}, y\right)^{1 / 3} \mathbb{1}_{F}(y) d y d x_{1} \\
& \leq 2 c(A) \int_{\mathscr{D}^{*}} \int_{\mathscr{D}_{x_{1}} \cap\left(-\infty, y_{1}\right)} J\left(x_{1}, y\right) \mathbb{1}_{F}(y) d y d x_{1} \\
& =2 c(A) \int_{\mathscr{D}^{*}} \int_{\widetilde{D}_{x_{1}} \cap\left(-\infty, y_{1}\right)} \mathbb{1}_{E}\left(x_{1}, y\right) d y d x_{1} \\
& =2 c(A)\left|E_{1}\right|
\end{aligned}
$$

Note that the second inequality follows from a simple modification of Lemma 2.1 in [11]. This finishes the proof.

Remark 9. A duality argument shows the following.
Corollary 10. Let $\varphi: \mathscr{D} \rightarrow \mathbb{R}$ be a $C^{2}$ function such that $\varphi_{12}^{\prime \prime}>0$. Suppose that there exists a constant $A$ such that, for each $y \in \mathscr{D}_{*}, \varphi_{12}^{\prime \prime}(\cdot, y) \in \mathscr{E}_{2}(A)$; that is,

$$
\varphi_{12}^{\prime \prime}\left(x_{1}, y\right)^{1 / 2} \varphi_{12}^{\prime \prime}\left(x_{2}, y\right)^{1 / 2} \leq A \varphi_{12}^{\prime \prime}\left(\frac{\left(x_{1}+x_{2}\right)}{2}, y_{1}\right)
$$

holds whenever $x_{1}<x_{2}$ and $\left[x_{1}, x_{2}\right] \subset \mathscr{D}^{y}$. Let $\mathscr{R}_{\varphi}$ be the operator given by (14). Then, there exists a constant $C$ that depends only on A such that

$$
\left\|\mathscr{R}_{\varphi} f\right\|_{L^{3}\left(\mathbb{R}^{2}\right)} \leq C\|f\|_{L^{3 / 2,3}\left(\mathbb{R}^{2}\right)}
$$

holds uniformly in $f \in C_{0}^{\infty}\left(\mathbb{R}^{2}\right)$.

Remark 11. As is well known, if $\mathscr{R}_{\varphi}$ maps boundedly from $L^{p, u}\left(\mathbb{R}^{2}\right)$ to $L^{q, v}\left(\mathbb{R}^{2}\right)$, then $(1 / p, 1 / q)$ belongs to the convex hull of $\{(0,0),(1,1),(2 / 3,1 / 3)\}$, and uniform estimates are possible only if $(1 / p, 1 / q)=(2 / 3,1 / 3)$. In the latter case, $3 / 2 \leq v \leq u \leq 3$ is necessary, implying the sharpness of the results. We refer interested readers to $[2,19]$.

\section{Acknowledgment}

This paper was completed with Ajou University Research Fellowship of 2011.

\section{References}

[1] J.-G. Bak, "An $L^{p}-L^{q}$ estimate for Radon transforms associated to polynomials," Duke Mathematical Journal, vol. 101, no. 2, pp. 259-269, 2000.

[2] J.-G. Bak, D. M. Oberlin, and A. Seeger, "Two endpoint bounds for generalized Radon transforms in the plane," Revista Matemática Iberoamericana, vol. 18, no. 1, pp. 231-247, 2002.

[3] S. H. Lee, "Remarks on the degenerate Radon transform in $R^{2}$," Proceedings of the American Mathematical Society, vol. 129, no. 11, pp. 3373-3378, 2001.

[4] S. Lee, "Endpoint $L^{p}-L^{q}$ estimates for degenerate Radon transforms in $R^{2}$ associated with real-analytic functions," Mathematische Zeitschrift, vol. 243, no. 2, pp. 217-241, 2003.

[5] S. Lee, "Endpoint $L^{p}-L^{q}$ estimates for some classes of degenerate Radon transforms in $R^{2}$," Mathematical Research Letters, vol. 11, no. 1, pp. 85-101, 2004.

[6] D. H. Phong and E. M. Stein, "Models of degenerate Fourier integral operators and Radon transforms," Annals of Mathematics, vol. 140, no. 3, pp. 703-722, 1994.

[7] A. Seeger, "Degenerate Fourier integral operators in the plane," Duke Mathematical Journal, vol. 71, no. 3, pp. 685-745, 1993.

[8] A. Seeger, "Radon transforms and finite type conditions," Journal of the American Mathematical Society, vol. 11, no. 4, pp. 869-897, 1998.

[9] C. W. Yang, " $L^{p}$ improving estimates for some classes of Radon transforms," Transactions of the American Mathematical Society, vol. 357, no. 10, pp. 3887-3903, 2005.

[10] Y. Choi, “Convolution estimates related to space curves," Journal of Inequalities and Applications, vol. 2011, no. 91, pp. 1-6, 2011.

[11] D. M. Oberlin, "Convolution with measures on flat curves in low dimensions," Journal of Functional Analysis, vol. 259, no. 7, pp. 1799-1815, 2010. 
[12] S. W. Drury, "Degenerate curves and harmonic analysis," Mathematical Proceedings of the Cambridge Philosophical Society, vol. 108, no. 1, pp. 89-96, 1990.

[13] J.-G. Bak, D. M. Oberlin, and A. Seeger, "Restriction of Fourier transforms to curves. II. Some classes with vanishing torsion," Journal of the Australian Mathematical Society, vol. 85, no. 1, pp. $1-28,2008$.

[14] D. M. Oberlin, "Convolution with affine arclength measures in the plane," Proceedings of the American Mathematical Society, vol. 127, no. 12, pp. 3591-3592, 1999.

[15] S. W. Drury and K. H. Guo, "Convolution estimates related to surfaces of half the ambient dimension," Mathematical Proceedings of the Cambridge Philosophical Society, vol. 110, no. 1, pp. 151-159, 1991.

[16] D. M. Oberlin, "Two estimates for curves in the plane," Proceedings of the American Mathematical Society, vol. 132, no. 11, pp. 3195-3201, 2004.

[17] M. Christ, "On the restriction of the Fourier transform to curves: endpoint results and the degenerate case," Transactions of the American Mathematical Society, vol. 287, no. 1, pp. 223238, 1985.

[18] D. M. Oberlin, "Convolution with measures on hypersurfaces," Mathematical Proceedings of the Cambridge Philosophical Society, vol. 129, no. 3, pp. 517-526, 2000.

[19] B. Stovall, "Endpoint bounds for a generalized Radon transform," Journal of the London Mathematical Society, vol. 80, no. 2, pp. 357-374, 2009. 


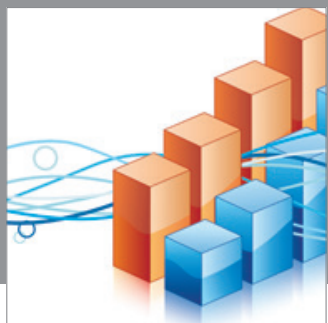

Advances in

Operations Research

mansans

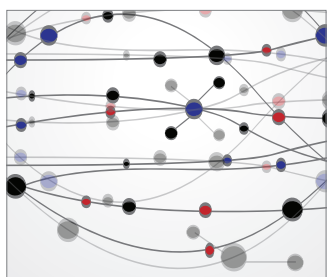

The Scientific World Journal
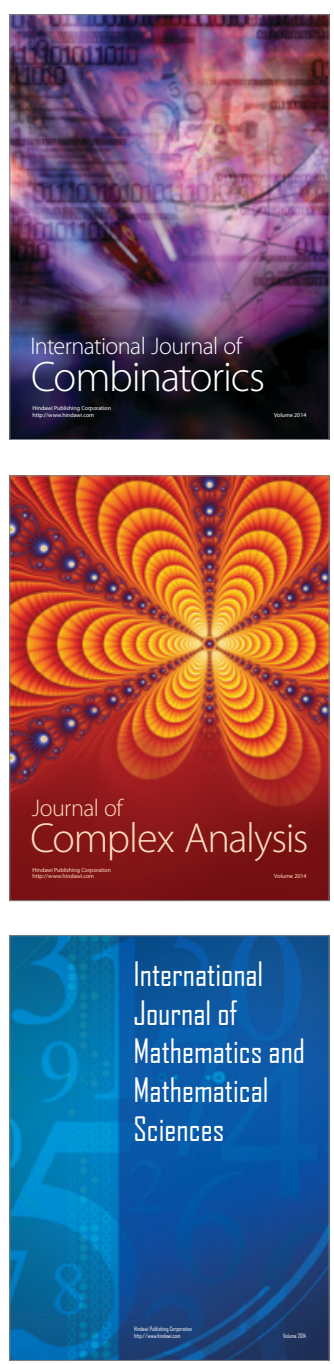
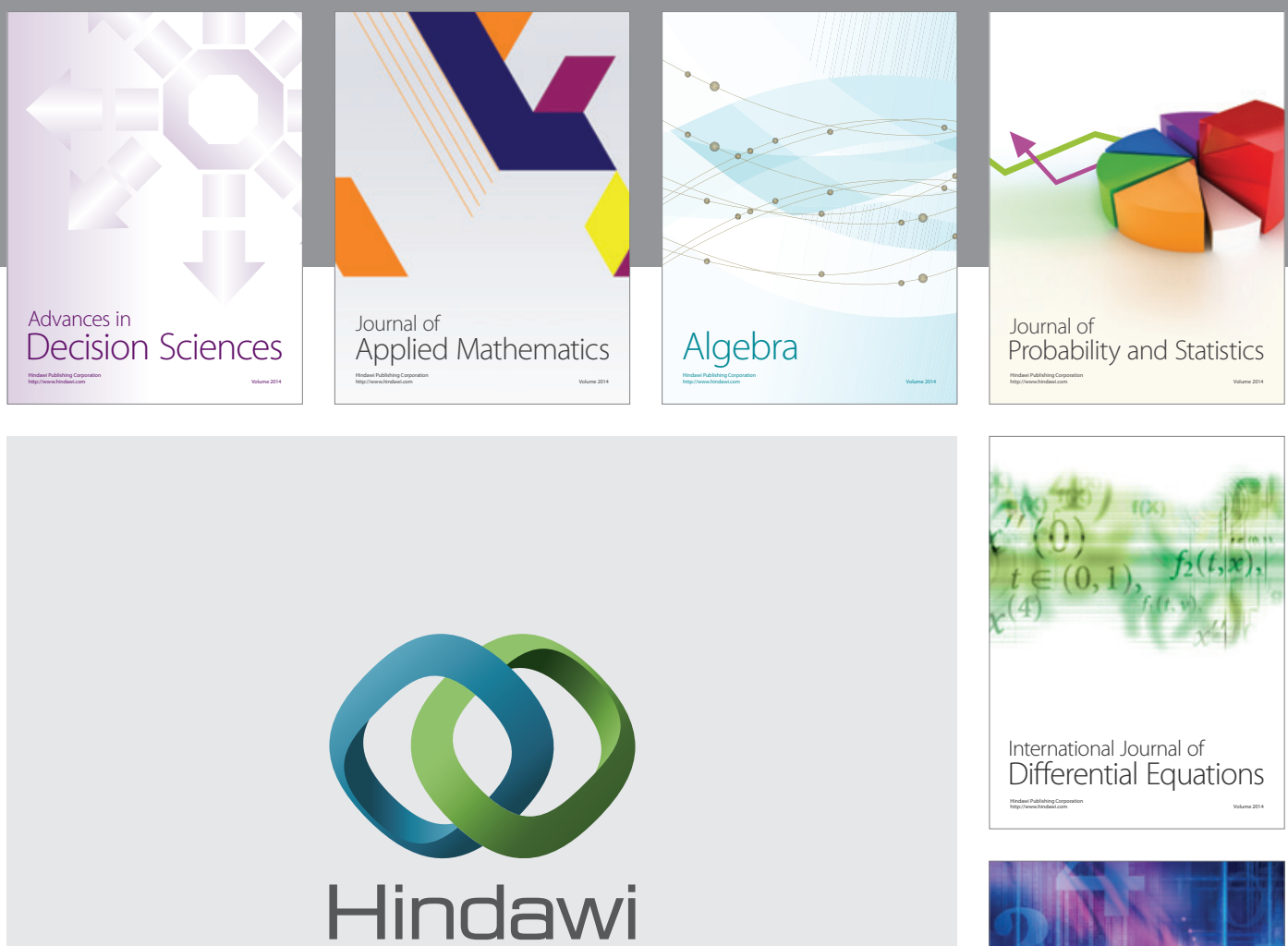

Submit your manuscripts at http://www.hindawi.com
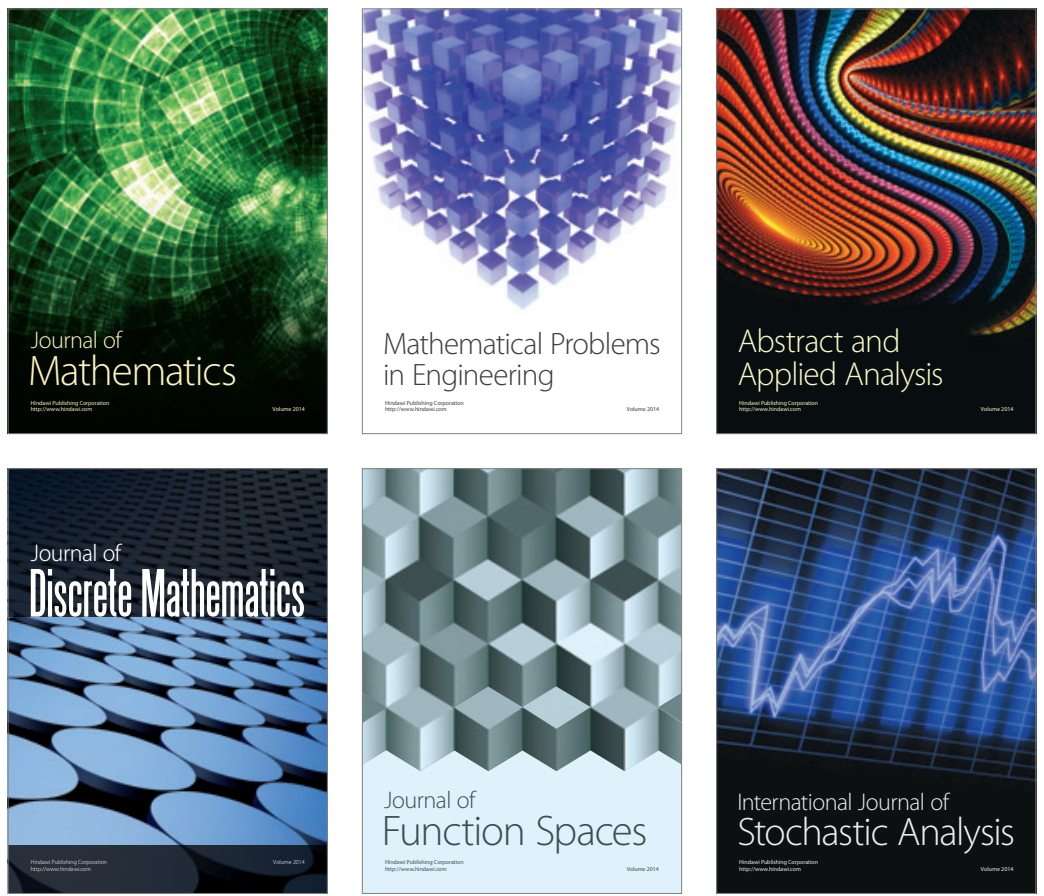

Journal of

Function Spaces

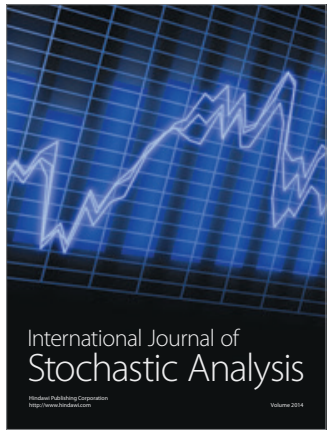

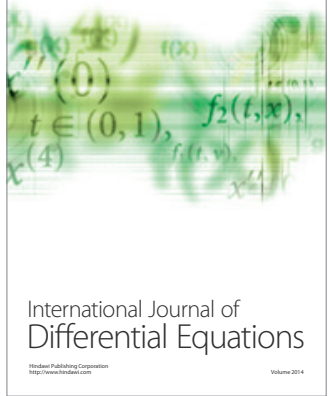
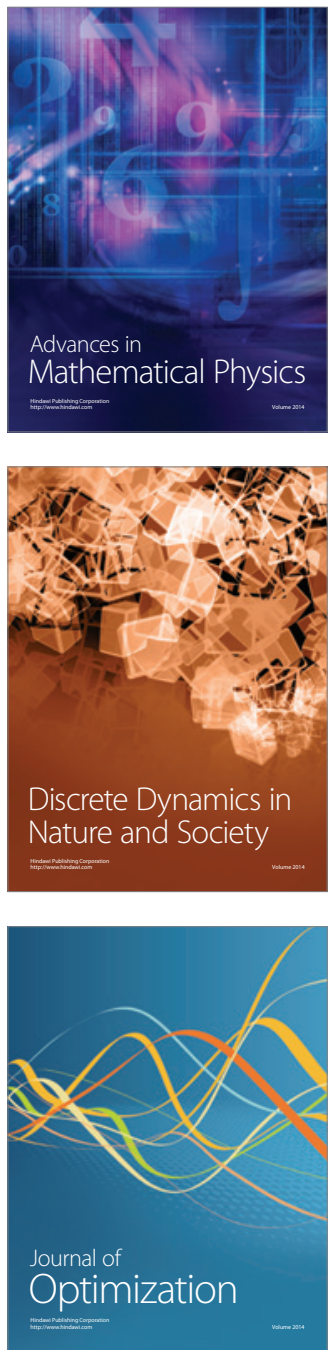